\title{
Left and right ventricular performance after arterial switch operation
}

\author{
Liselotte M. Klitsie, MSc, ${ }^{a}$ Arno A. W. Roest, MD, PhD, ${ }^{a}$ Irene M. Kuipers, MD, PhD, ${ }^{b}$ \\ Mark G. Hazekamp, MD, PhD, ${ }^{c}$ Nico A. Blom, MD, PhD, ${ }^{a}$ and Arend D. J. Ten Harkel, MD, PhD ${ }^{\mathrm{a}}$
}

Objective: Recent descriptions of decreased exercise capacity 10 to 15 years after arterial switch operation (ASO) suggest subclinical hemodynamic restrictions. Persistent impairment of ventricular performance following ASO may add to this. We aimed to characterize the time course of changes in biventricular performance within the first year following ASO.

Methods: We prospectively included 26 patients with transposition of the great arteries undergoing ASO and 20 age-matched controls. Left and right ventricular systolic and diastolic performance was assessed using tissue Doppler imaging-derived peak systolic velocity, peak diastolic velocity, and peak early wave Doppler flow velocity/early diastolic tissue Doppler imaging velocity as well as mitral and tricuspid annular plane systolic excursion. Furthermore, left ventricular longitudinal, radial, and circumferential strain were assessed using speckle tracking strain imaging. Studies were performed preoperatively, 1 day postoperatively, at discharge, and at medium-term follow-up (9 months [interquartile range, 6-23 months] postoperatively).

Results: After an initial decrease in biventricular systolic and diastolic performance 1 day postoperatively versus preoperatively, recovery was observed in all parameters during medium-term follow-up. At mediumterm follow-up left ventricular systolic and diastolic performance parameters were comparable in patients and controls. In contrast, right ventricular systolic and diastolic performance were still impaired in patients versus controls roughly 1 year postoperatively (tricuspid annular plane systolic excursion, $11.6 \pm 2.2$ vs $18.6 \pm 3.1 \mathrm{~mm}$; right ventricular peak systolic velocity, $8.1 \pm 2.3$ vs $12.6 \pm 1.8 \mathrm{~cm} / \mathrm{second}$; right ventricular peak diastolic velocity, $12.4 \pm 3.0$ vs $18.2 \pm 4.2 \mathrm{~cm} / \mathrm{second}$; and right ventricular peak early wave Doppler flow velocity/early diastolic tissue Doppler imaging velocity, $6.7 \pm 2.1$ vs $4.3 \pm 1.3$; all $P$ s $<.001$ ).

Conclusions: If early ASO is performed, left ventricular performance recovers to control values within the first postoperative year. In contrast, right ventricular systolic and diastolic performance remained impaired during follow-up, which stresses the importance of postoperative follow-up of right ventricular performance. (J Thorac Cardiovasc Surg 2014;147:1561-7)

Earn CME credits at

http://jtcvs.com/cme/home

Since the introduction of anatomical correction of transposition of the great arteries (TGA) with the arterial switch operation (ASO), patient survival has improved. In combination with ongoing refinements of the surgical technique, this has

\footnotetext{
From the Departments of Pediatric Cardiology ${ }^{\mathrm{a}}$ and Cardiothoracic Surgery, ${ }^{\mathrm{c}}$ Leiden University Medical Center, Leiden, The Netherlands; and Department of Pediatric

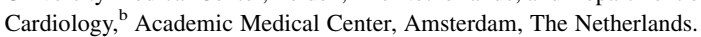

L.M.K. is supported by a grant from the MD/PhD project of Leiden University Medical Center, Leiden, The Netherlands. The sponsor had no role in the design of the study, the collection of data, or the writing and submission of this manuscript.

Disclosures: Authors have nothing to disclose with regard to commercial support.

Received for publication May 17, 2013; revisions received July 2, 2013; accepted for publication July 12, 2013; available ahead of print Sept 16, 2013.

Address for reprints: Arend D. J. Ten Harkel, MD, Department of Pediatric Cardiology, Leiden University Medical Center, PO Box 9600, Room J6S-208, 2300 RC Leiden, The Netherlands (E-mail: A.D.J.Ten_Harkel@lumc.nl). 0022-5223/\$36.00

Copyright (c) 2014 by The American Association for Thoracic Surgery http://dx.doi.org/10.1016/j.jtcvs.2013.07.048
}

lead to low surgical mortality ${ }^{1}$ and good long-term survival of patients undergoing ASO in the current era. ${ }^{2}$ Nonetheless, recent descriptions of decreased exercise capacity 10 to 15 years after ASO suggest that subclinical hemodynamic restrictions remain. ${ }^{3,4}$ Hemodynamic impairment could develop over time as a consequence of common residua and complications of ASO, including pulmonary artery obstruction and coronary artery problems. ${ }^{4}$ However, persistent impairment of cardiac performance following surgical correction may also play a role.

Persistent impairment of cardiac performance has been previously described in pediatric patients after surgical correction of a congenital heart defect using cardiopulmonary bypass $(\mathrm{CPB}){ }^{5}$ In patients born with TGA, decreased left ventricular (LV) performance has been described within the first 48 hours following ASO. ${ }^{6,7}$ However, to our knowledge, continuing follow-up of the changes evoked by surgery and the time course and extent of recovery is limited. Furthermore, right ventricular (RV) performance is underexposed in most studies.

With the advent of echocardiographic techniques, including tissue Doppler imaging (TDI) and speckle tracking 


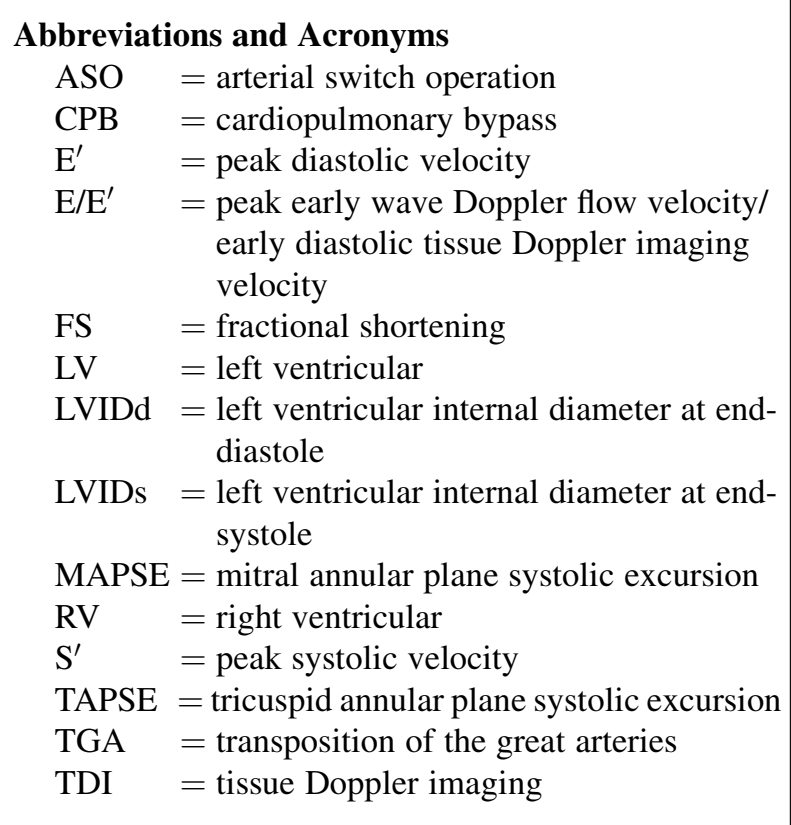

strain imaging, the detection of subtle impairment of biventricular systolic and diastolic performance has been greatly enhanced. ${ }^{8}$ Additionally, these techniques offer the advantage of regional assessment of myocardial performance. Accordingly, in our study we aimed to characterize the time course of changes in biventricular performance within the first year following ASO using TDI and speckle tracking strain imaging.

\section{METHODS}

We prospectively enrolled all consecutive patients with simple TGA, with or without a ventricular septal defect, who underwent ASO at our institution between 2009 and 2012. Patients with major additional defects, including Taussig-Bing anomaly and aortic arch pathology, were excluded. Additionally, 1 group of healthy children, who were referred to our institution with an asymptomatic, innocent heart murmur, were included as controls. This group was age-matched to patients at medium-term follow-up. The institutional review board at our institution approved this study and written informed consent was obtained from the parents.

Surgical correction was carried out via a median sternotomy using highflow, moderate hypothermic CPB with bicaval cannulation. Before crossclamping, the open ductus arteriosus was ligated. Subsequently the aorta was crossclamped and cold crystalloid cardioplegia was infused; this was repeated every 30 minutes. The operation included dissection of the aorta and pulmonary artery above the level of the commisures, transplantation of the coronary arteries from the aortic sinus into the corresponding pulmonary sinus using the coronary artery-button technique, switching of the aorta and pulmonary artery by the Lecompte manoeuvre whenever possible, and reconstruction of the neopulmonary trunk with an autologous pericardial patch.

Demographic parameters, including weight, body surface area, sex, and age were documented at study inclusion. Body surface area in square meters was calculated using Boyd's formula $\left(0.0004688 \times\right.$ weight $\left.^{(0.8168-0.0154 \times \log [\text { weightweight] })}\right)$ using weight in grams. Additionally, operative parameters were collected, including CPB time and aortic crossclamp time.
All patients and controls underwent transthoracic echocardiography, without sedation, to comprehensively assess biventricular systolic and diastolic performance. In patients examinations were performed preoperatively, 1 day postoperatively, at hospital discharge, and at a medium-term follow-up of roughly 1 year postoperatively. Subsequently changes in echocardiographic parameters in patients during follow-up were evaluated. Controls underwent only 1 examination, and were compared with patients at medium-term follow-up.

\section{Echocardiography}

Transthoracic echocardiography was performed to assess biventricular performance using a commercially available system (Vivid-7.0.0; General Electric Vingmed Ultrasound, Horten, Norway). Furthermore, the presence and severity of pulmonary artery obstruction at discharge was assessed using continuous Doppler flow velocity in the parasternal short-axis view. The maximal peak flow velocity in the main pulmonary artery or pulmonary artery branches was used as the maximal pulmonary artery velocity. ${ }^{4}$ Images were stored in digital format to allow off-line analyses using EchoPac version 11.1.8 (General Electric Vingmed Ultrasound). Off-line analysis was performed by 1 observer (L.M.K.) to limit possible interobserver variability. Subsequently, results were reviewed and discussed with a second observer (A.D.J.H.). Patients without sinus rhythm at the time of echocardiographic investigation were excluded.

\section{M-Mode}

Fractional shortening (FS) was calculated from M-mode recordings of the LV long axis to assess LV systolic performance. To calculate FS, LV internal diameter at end-diastole (LVIDd) and LV internal diameter at end-systole (LVIDs) were assessed in millimeters and combined as follows ((LVIDd-LVIDs) $/$ LVIDd $) \times 100 \%$.

In M-mode recordings of the apical 4-chamber view LV and RV systolic performance was assessed using measurements of respectively mitral annular plane systolic excursion (MAPSE) and tricuspid annular plane systolic excursion (TAPSE). The cursor was placed at the mitral or tricuspid annulus free wall, as previously described. ${ }^{9}$ Subsequently the maximal excursion of the valve plane was assessed from end-diastole to end-systole.

\section{TDI}

Biventricular systolic and diastolic performance was characterized using pulsed-wave TDI. TDI images were obtained in 2-dimensional images of the 4-chamber view throughout 3 consecutive cardiac cycles. The angle of insonation was adjusted to align the ultrasound beam along the direction of myocardial motion. Subsequently, myocardial velocity curves were acquired by placing the cursor at the basal part of the LV lateral wall and RV free wall. In each curve, peak systolic velocities $\left(S^{\prime}\right)$ and peak early diastolic velocities $\left(\mathrm{E}^{\prime}\right)$ were assessed. In addition, peak early wave Doppler flow velocity/early diastolic tissue Doppler imaging velocity $\left(\mathrm{E} / \mathrm{E}^{\prime}\right)$, a diastolic parameter strongly correlated with ventricular filling pressure, ${ }^{10}$ was calculated to assess diastolic performance. LV and RV E were assessed by measurements of peak early wave velocity (centimeters per second) in spectral Doppler tracings recorded in the apical 4-chamber view at the tip of the mitral and tricuspid valve.

\section{Speckle Tracking Strain Imaging}

In addition to TDI, LV systolic performance was evaluated using speckle tracking strain analyses performed in grayscale images of the apical 4-chamber view (longitudinal analysis) and the LV parasternal shortaxis view (radial and circumferential analysis). Images were obtained with optimized sector width and frame rate (preferably 60-90 frames/second). In these images, manual endocardial border tracing at end-systole was used to set the region of interest. The region of interest was automatically divided into 6 segments. In the 4-chamber view this included the 
TABLE 1. Demographic parameters and operation characteristics of patients with transposition of the great arteries

\begin{tabular}{lc}
\hline \multicolumn{1}{c}{ Demographic parameter } & Total \\
\hline $\mathrm{N}$ & 26 \\
Male, $\mathrm{n}(\%)$ & $16(62)$ \\
Age at surgery, $\mathrm{d}^{*}$ & $10(7-14)$ \\
Weight, $\mathrm{kg}$ & $3.5 \pm 0.5$ \\
Body surface area, $\mathrm{m}^{2}$ & $0.23 \pm 0.02$ \\
Cardiopulmonary bypass time, min & $160 \pm 33$ \\
Aortic crossclamp time, min & $106 \pm 25$ \\
Maximal pulmonary artery velocity, $\mathrm{m} / \mathrm{s}$ & $2.6 \pm 0.9$ \\
\hline
\end{tabular}

Approximately normally distributed data are expressed as mean \pm standard deviation. *Values expressed as median (interquartile range)

basal, mid, and apical segments of the LV lateral wall and the interventricular septum. The short-axis image was divided into a septal, anteroseptal, anterior, lateral, posterior, and inferior segment to evaluate both radial and circumferential strain. In each segment tracking quality was automatically evaluated and this resulted in automatic rejection or acceptation of the segment. Although an observer could override this automatic decision based on visual evaluation, ${ }^{11}$ this was used very conservatively. ${ }^{12}$ Data obtained by speckle tracking was displayed in longitudinal time-strain curves for each segment in the 4-chamber view and radial and circumferential time-strain curves for each segment in the short-axis image. ${ }^{13}$ From these time-strain curves segmental peak strain was obtained, defined as the most negative longitudinal or circumferential and most positive radial strain value, respectively, at any time point during 1 cardiac cycle. Finally, the mean strain of the 6 segments in 1 view was calculated and constituted global peak strain. ${ }^{12}$

\section{Statistics}

Approximately normally distributed data are expressed as mean \pm standard deviation. Not approximately normally distributed data are expressed as median (interquartile range). The Wilcoxon signed rank test was used to assess differences in biventricular performance in patients 1 day postoperatively versus preoperatively, 1 year postoperatively versus 1 day postoperatively, and 1 year postoperatively versus preoperatively. Because considerable variation in timing of medium-term follow-up was observed, linear mixed models were constructed for each echocardiographic parameter to assess the influence of follow-up duration. The duration between medium-term follow-up and the operation date was included as an independent variable in these models.

The Mann-Whitney $U$ test was used to assess differences between controls and patients at medium-term follow-up, between patients with and without significant pulmonary artery stenosis (maximal pulmonary artery velocity $>2.5 \mathrm{~m} / \mathrm{second}$ ), and between patients with and without abnormal coronary anatomy. Correlations between RV performance parameters and maximal pulmonary artery velocity at discharge were assessed using scatter plots and Spearman's rank test. Finally, scatter plots and Spearman's rank test were used to assess the correlation between RV performance parameters and aortic crossclamp time and CPB time at medium-term follow-up, and between age at operation and RV performance parameters preoperatively and at medium-term follow-up. Data analysis was performed using SPSS 20 (IBM-SPSS Inc, Armonk, NY).

\section{RESULTS}

A total of 27 patients with TGA were enrolled. One patient, who underwent reoperation for pulmonary artery stenosis before hospital discharge, was excluded. Accordingly, 26 patients were included in our study. Additionally, a group of 20 age-matched controls were included. No differences were observed between patients and controls in weight $(8.5 \pm 2.2$ vs $9.6 \pm 2.6 \mathrm{~kg} ; P=.16)$.

Demographic characteristics of patients are summarized in Table 1. Before surgery, 18 of 26 patients underwent a balloon atrial septostomy. The Lecompte procedure was carried out in all patients. Furthermore, in 7 patients a ventricular septal defect was closed (4 patch, 3 direct suture) in addition to ASO. In 2 patients the patch was visible in the 4chamber view and echocardiograms of these patients were excluded from longitudinal speckle tracking strain analysis.

In 18 of 26 patients coronary anatomy was normal (1LCx-2R; R, right coronary artery; L, left coronary artery; $\mathrm{Cx}$, circumflex coronary artery; 1 , first coronary sinus; 2 , second coronary sinus). The remaining 8 patients had a differing coronary anatomy, including 4 patients with $1 \mathrm{~L}-2 \mathrm{RCx}$ and 1 patient each with $1 \mathrm{R}-2 \mathrm{LCx}, 1 \mathrm{Cx}-2 \mathrm{RL}$, $1 \mathrm{LCx}-2 \mathrm{RCx}$, and $2 \mathrm{RCxL}$. In 1 of these patients, the coronary artery was damaged and subsequently repaired during surgery, without evidence of postoperative ischemia. No differences were observed in aortic crossclamp time or CPB time between patients with normal versus differing coronary artery anatomy. One patient was reoperated for pulmonary artery stenosis after hospital discharge. The echocardiogram at medium-term follow-up in this patient was not included. Finally none of the patients had more than a trace of aortic insufficiency at medium-term follow-up, as qualitatively assessed using echocardiography.

In the patients born with TGA, echocardiography was performed preoperatively $(\mathrm{n}=25), 1$ day postoperatively $(\mathrm{n}=22)$, at discharge (9 days [interquartile range, 7-12 days] postoperatively $),(n=25)$, and after medium-term follow-up (9 months [interquartile range, 6-23 months] postoperatively) $(\mathrm{N}=20)$. In none of the linear mixed models a contribution of the duration of follow-up was observed, except in the MAPSE model. Because MAPSE values were comparable to controls at medium-term follow-up, as described below, this has little influence on our conclusions. Therefore we consider the medium-term follow-up measurement to be time-independent.

\section{LV}

In patients with TGA, LV chamber sizes were normal preoperatively (LVIDd, $19 \pm 3 \mathrm{~mm}$; LVIDs, $11 \pm 2 \mathrm{~mm}$ ). ${ }^{14}$ Follow-up of parameters assessing LV systolic performance including FS, MAPSE, LV S', and LV global peak strain parameters revealed a decrease in these parameters 1 day postoperatively versus preoperatively (see Tables 2 and 3 and Figure 1). Subsequently, a recovery was observed in LV systolic performance parameters during medium-term followup. This resulted in comparable values of all parameters describing LV systolic performance in patients versus controls 1 year postoperatively (see Tables 2 and 3 and Figure 1).

In addition to global peak strain parameters, segmental peak strain parameters were compared in patients at 
TABLE 2. M-mode and tissue Doppler imaging parameters in patients preoperatively and during postoperative follow-up and in controls

\begin{tabular}{|c|c|c|c|c|c|c|c|c|c|}
\hline & \multicolumn{4}{|c|}{ Patients } & \multirow[b]{2}{*}{ Controls } & \multirow{2}{*}{$\begin{array}{c}P \text { value } \\
\text { preop vs } \\
1 \text { d postop }\end{array}$} & \multirow{2}{*}{$\begin{array}{c}P \text { value } \\
1 \text { d postop vs } \\
\text { medium-term* }\end{array}$} & \multirow{2}{*}{$\begin{array}{c}P \text { value } \\
\text { preop vs } \\
\text { medium-term* }\end{array}$} & \multirow{2}{*}{$\begin{array}{c}P \text { value patients } \\
\text { medium-term* vs } \\
\text { controls }\end{array}$} \\
\hline & Preop & $\begin{array}{c}1 \mathrm{~d} \\
\text { Postop }\end{array}$ & Discharge & $\begin{array}{l}\text { Medium-term } \\
\text { follow-up }\end{array}$ & & & & & \\
\hline \multicolumn{10}{|l|}{ Left ventricle } \\
\hline FS, $\%$ & $40.2 \pm 8.1$ & $30.1 \pm 7.1$ & $37.4 \pm 8.3$ & $37.5 \pm 5.4$ & $39.8 \pm 4.5$ & .007 & .06 & .21 & .14 \\
\hline MAPSE, mm & $7.4 \pm 1.4$ & $4.6 \pm 1.3$ & $5.7 \pm 1.2$ & $9.8 \pm 1.6$ & $10.7 \pm 1.6$ & .001 & .002 & .001 & .07 \\
\hline $\mathrm{S}^{\prime}, \mathrm{cm} / \mathrm{sec}$ & $6.5 \pm 2.1$ & $4.1 \pm 1.1$ & $4.0 \pm 1.4$ & $6.5 \pm 1.3$ & $6.2 \pm 1.3$ & .002 & .001 & .90 & .62 \\
\hline $\mathrm{E}^{\prime}, \mathrm{cm} / \mathrm{sec}$ & $8.3 \pm 3.5$ & $5.5 \pm 2.4$ & $6.7 \pm 2.9$ & $12.6 \pm 2.9$ & $13.6 \pm 3.6$ & .005 & .001 & .001 & .36 \\
\hline $\mathrm{E} / \mathrm{E}^{\prime}$ & $16.7 \pm 10.4$ & $12.4 \pm 5.5$ & $13.9 \pm 6.3$ & $9.0 \pm 2.9$ & $9.0 \pm 3.4$ & .33 & .06 & .001 & .80 \\
\hline \multicolumn{10}{|l|}{ Right ventricle } \\
\hline TAPSE, mm & $10.2 \pm 1.8$ & $3.5 \pm 1.1$ & $5.5 \pm 1.2$ & $11.6 \pm 2.2$ & $18.6 \pm 3.1$ & $<.001$ & .001 & .007 & $<.001$ \\
\hline $\mathrm{S}^{\prime}, \mathrm{cm} / \mathrm{sec}$ & $10.1 \pm 2.5$ & $3.2 \pm 1.3$ & $3.9 \pm 1.2$ & $8.1 \pm 2.3$ & $12.6 \pm 1.8$ & $<.001$ & .001 & .048 & $<.001$ \\
\hline $\mathrm{E}^{\prime}, \mathrm{cm} / \mathrm{sec}$ & $16.5 \pm 4.0$ & $3.8 \pm 1.4$ & $6.4 \pm 1.8$ & $12.4 \pm 3.0$ & $18.2 \pm 4.2$ & $<.001$ & .001 & .022 & $<.001$ \\
\hline $\mathrm{E} / \mathrm{E}^{\prime}$ & $6.3 \pm 1.5$ & $16.3 \pm 8.1$ & $10.3 \pm 4.0$ & $6.7 \pm 2.1$ & $4.3 \pm 1.3$ & $<.001$ & .001 & .57 & $<.001$ \\
\hline
\end{tabular}

Values are expressed as mean \pm standard deviation. Preop, Preoperatively; postop, postoperatively; FS, fractional shortening; MAPSE, mitral annular plane systolic excursion; $S^{\prime}$, peak systolic tissue Doppler imaging velocity; $E^{\prime}$, peak early diastolic tissue Doppler imaging velocity; $E / E^{\prime}$, peak early wave Doppler flow velocity/early diastolic tissue Doppler imaging velocity; TAPSE, tricuspid annular plane systolic excursion. *Medium-term follow-up was 9 months (interquartile range, 6-23 months) postoperatively.

medium-term follow-up versus controls. These analyses yielded comparable peak strain values in most segments. However, circumferential peak strain in the septal and anteroseptal segment remained impaired in patients versus controls (septal: $-24.6 \% \pm 5.3 \%$ vs $-31.4 \% \pm 7.0 \%$; $P=.008$; anteroseptal: $-26.2 \% \pm 8.0 \%$ vs $-32.3 \% \pm$ $6.7 \% ; P=.04)$ and longitudinal peak strain in the basal septal segment remained increased $(-21.6 \% \pm 4.4 \%$ vs $-18.3 \% \pm 3.7 \% ; P=.02$ ) (see Figure 2).

$\mathrm{LV}$ diastolic performance, as assessed using $\mathrm{LV} \mathrm{E}^{\prime}$, was decreased 1 day postoperatively versus preoperatively (5.5 \pm 2.4 vs $8.3 \pm 3.5 \mathrm{~cm} /$ second; $P=.005$ ). Subsequent follow-up revealed an increase in $\mathrm{LV} \mathrm{E}^{\prime}(5.5 \pm 2.4$ vs $12.6 \pm 2.9 \mathrm{~cm} / \mathrm{second} ; P=.001$ ) during the first postoperative year. At medium-term follow-up, no differences were observed in LV diastolic performance parameters in patients versus controls (Table 2).

\section{RV}

Both parameters describing RV systolic performance, including RV $\mathrm{S}^{\prime}$ and TAPSE, and parameters describing $\mathrm{RV}$ diastolic performance, including $\mathrm{RV} \mathrm{E}^{\prime}$ and $\mathrm{RV} \mathrm{E} / \mathrm{E}^{\prime}$, were impaired 1 day postoperatively versus preoperatively (see Table 2 and Figure 1). Subsequently, a recovery was observed in all RV performance parameters during medium-term follow-up (see Table 2 and Figure 1).
However, in contrast to LV performance parameters, parameters describing RV systolic and diastolic performance were still impaired in patients versus controls roughly 1 year postoperatively (TAPSE, $11.6 \pm 2.2$ vs $18.6 \pm 3.1$ $\mathrm{mm}$; RV S', $8.1 \pm 2.3$ vs $12.6 \pm 1.8 \mathrm{~cm} / \mathrm{second}^{\prime} \mathrm{RV} \mathrm{E}^{\prime}$, $12.4 \pm 3.0$ vs $18.2 \pm 4.2 \mathrm{~cm} /$ second; $\mathrm{RV}$ E/E' $6.7 \pm 2.1$ vs $4.3 \pm 1.3$; all $P$ s $<.001)$. Furthermore both RV $\mathrm{S}^{\prime}$ and $\mathrm{E}^{\prime}$ were lower in patients at medium-term follow-up versus preoperatively ( $\mathrm{RV} \mathrm{S}^{\prime}: 8.1 \pm 2.3$ vs $10.1 \pm 2.5 \mathrm{~cm} /$ second; $P=.048 ; \mathrm{RV} \mathrm{E}^{\prime}: 12.4 \pm 3.0$ vs $16.5 \pm 4.0 \mathrm{~cm} / \mathrm{second}$; $P=.022)$.

No correlation was observed between aortic crossclamp time or CPB time and RV performance parameters at medium-term follow-up. Furthermore, age at operation was not correlated to RV performance parameters preoperatively or at medium-term follow-up, except for a weak correlation at medium-term follow-up between TAPSE and age at operation $\left(P=.016 ; R^{2}=0.28\right)$.

In 4 patients tricuspid regurgitation velocity was $>2.5 \mathrm{~m} /$ second. No significant correlation was observed between maximal pulmonary artery velocity at discharge and parameters describing RV systolic and diastolic performance at discharge or medium-term follow-up, except for a weak correlation between maximal pulmonary artery velocity and $\mathrm{RV} \mathrm{E}^{\prime}$ at discharge $\left(P=.05 ; R^{2}=0.17\right)$. Furthermore, at discharge no differences were observed in RV

TABLE 3. Left ventricular speckle tracking strain derived parameters in patients preoperatively and during postoperative follow-up and in controls

\begin{tabular}{|c|c|c|c|c|c|c|c|c|c|}
\hline \multirow[b]{2}{*}{$\begin{array}{c}\text { Global peak } \\
\text { strain, } \%\end{array}$} & \multicolumn{4}{|c|}{ Patients } & \multirow[b]{2}{*}{ Controls } & \multirow{2}{*}{$\begin{array}{c}P \text { value } \\
\text { preop vs } \\
1 \text { d postop }\end{array}$} & \multirow{2}{*}{$\begin{array}{c}P \text { value } \\
1 \text { d postop vs } \\
\text { medium-term* }\end{array}$} & \multirow{2}{*}{$\begin{array}{c}P \text { value } \\
\text { preop vs } \\
\text { medium-term* }\end{array}$} & \multirow{2}{*}{$\begin{array}{l}P \text { value patients } \\
\text { medium-term* vs } \\
\text { controls }\end{array}$} \\
\hline & Preop & 1 d Postop & Discharge & $\begin{array}{c}\text { Medium-term } \\
\text { follow-up }\end{array}$ & & & & & \\
\hline Longitudinal & $-21.7 \pm 3.4$ & $-13.7 \pm 3.9$ & $-16.4 \pm 3.2$ & $-21.5 \pm 3.6$ & $-23.1 \pm 3.3$ & $<.001$ & .007 & .83 & .14 \\
\hline Radial & $42.4 \pm 14.6$ & $22.3 \pm 9.5$ & $27.1 \pm 10.6$ & $40.3 \pm 12.0$ & $46.5 \pm 12.9$ & .001 & .005 & .68 & .13 \\
\hline Circumferential & $-22.9 \pm 4.6$ & $-16.8 \pm 4.2$ & $-19.6 \pm 6.0$ & $-21.1 \pm 4.2$ & $-24.0 \pm 4.3$ & .003 & .01 & .036 & .13 \\
\hline
\end{tabular}

Values are expressed as mean \pm standard deviation. Preop, Preoperatively; postop, postoperatively. *Medium-term follow-up was 9 months (interqaurtile range, 6-23 months) postoperatively. 

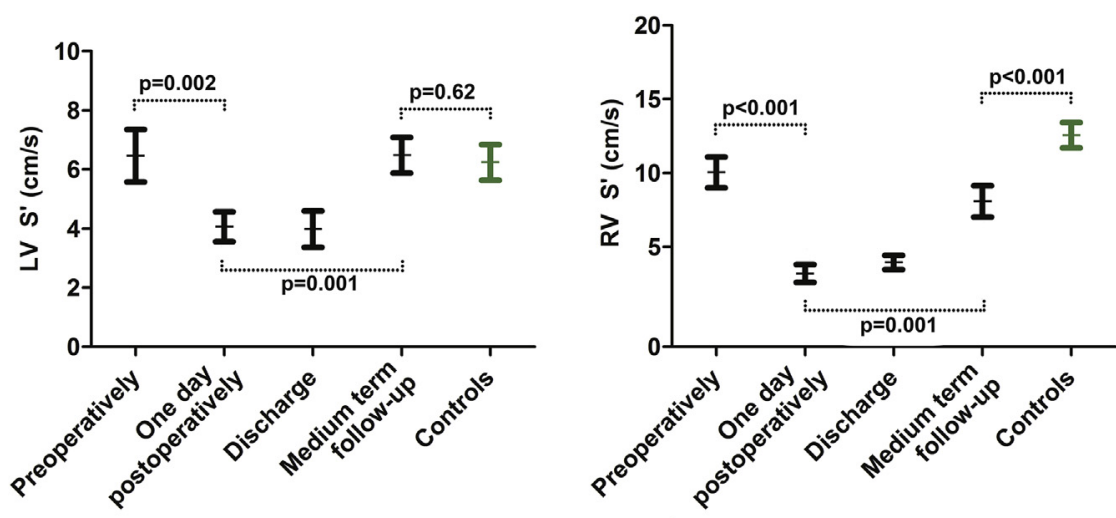

FIGURE 1. Box plots depicting mean and $95 \%$ confidence interval of the mean of left ventricular $(L V)$ systolic and right ventricular $(R V)$ systolic tissue Doppler imaging parameters in patients during follow-up and in controls. $S^{\prime}$, Peak systolic tissue Doppler imaging velocity.

performance parameters between patients with and without significant pulmonary stenosis.

\section{DISCUSSION}

Follow-up in patients born with TGA after ASO is still characterized by decreased exercise capacity. ${ }^{3,4}$ Hemodynamic restrictions, including impaired ventricular performance, may add to this. Our results, which describe a decrease in biventricular performance parameters directly postoperatively, emphasize that ventricular performance decreases after surgery. Furthermore, our study highlights that although LV performance recovers to control values, RV systolic and diastolic performance remain impaired within the first postoperative year.

\section{LV}

The acute decrease in LV systolic and diastolic performance directly following surgery observed in our study is in agreement with previous studies of LV performance within the first 48 hours following ASO. ${ }^{6,7}$ The cause of this decrease could be multifactorial, including altered

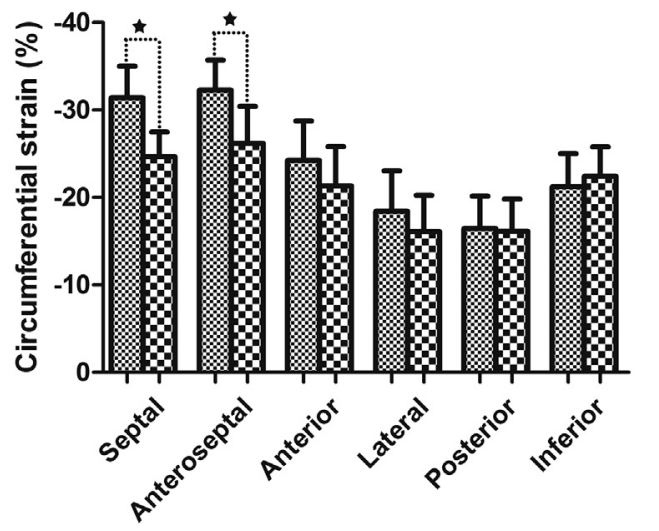

FIGURE 2. Segmental circumferential peak strain in patients born with transposition of the great arteries versus controls. Bar graph depicts mean and $95 \%$ confidence interval of the mean peak circumferential segmental strain. *Segments in which a significant difference was observed between patients and age-matched controls at medium-term follow-up. loading conditions, direct surgical trauma, reoxygenation injury, and CPB-associated ischemia or inflammatory cascades. ${ }^{6,7,15,16}$ Especially the altered loading conditions have been suggested to play a role in inducing LV dysfunction directly postoperatively. The LV is confronted with an acute increase in afterload following ASO because the ventricle faces pumping against the high vascular resistance of the systemic circulation. ${ }^{6}$ This afterload increase may induce a decrease in LV performance. Yet, care must be taken in interpreting these results, because both TDI parameters and strain parameters are possibly loaddependent. $^{17}$

Follow-up studies beyond the first 48 hours have merely focused on long-term follow-up. These studies have presented contrasting results. ${ }^{18-22}$ Colan and colleagues ${ }^{20}$ described normal FS 0.5 to 10 years postoperatively. In contrast, decreased FS and ejection fraction were described in more recent studies in patients versus controls 10 to 15 years postoperatively. ${ }^{18,19}$ Our results, which portray an improvement in LV performance parameters after surgery to control values, suggest total recovery of $\mathrm{LV}$ performance within the first postoperative year. Future studies are necessary to exclude subsequent deterioration.

Of note is that although assessment of LV global peak strain parameters revealed no differences between patients and controls 1 year postoperatively, circumferential peak strain in the septal and anteroseptal segment did remain impaired. Previously, using wall motion analyses and myocardial perfusion scans, segmental abnormalities have also been observed. ${ }^{18,23}$ These abnormalities presented in variable segments. Yet, a trend of abnormal motion in especially the anterior segment can be observed. ${ }^{18,23}$ Furthermore, basal rotation was described to be decreased in patients born with TGA versus controls 12 years postoperatively; this was most pronounced in the anterior segment. ${ }^{21}$ In combination with our results this could imply increased vulnerability of anterior segments. However this suggestion must be interpreted with caution, considering the variability of techniques and lack of specific analysis. 


\section{RV}

Studies describing follow-up of ASO have generally focused on the LV. Yet our results support increased awareness of RV performance, because both RV systolic and diastolic performance parameters do not completely recover to preoperative levels. Furthermore, all RV performance parameters remained impaired in patients versus controls up to 1 year postoperatively. Although our results are unique in their description of RV performance within the first postoperative year, they are largely in line with previous longterm follow-up studies. Whereas 1 study described normal $\mathrm{RV}$ ejection fraction 10 years after $\mathrm{ASO},{ }^{24}$ most other longterm follow-up studies have demonstrated a variable degree of impairment of RV systolic ${ }^{21,25}$ and diastolic ${ }^{26}$ performance. The persistent impairment in RV performance parameters is of interest because subtle changes in RV TDI parameters have shown to be of prognostic value for increased risk of cardiovascular events. ${ }^{27,28}$ Thus, our study identifies an increased vulnerability, which is already present directly after ASO. This impairment may not have a direct clinical consequence. Yet, if it persists, its prognostic value warrants careful follow-up of RV performance and may possibly contribute to earlier start of therapeutic strategies.

Several theories could explain the persistent impairment of especially RV performance following ASO. Preoperatively, possible ischemia and hypoxia may have affected cardiac performance and leave the heart more vulnerable for reoxygenation injury during surgery. ${ }^{16}$

Perioperative factors that may have contributed include incomplete myocardial protection, $\mathrm{CPB}$, and pericardial incision. ${ }^{29-31}$ The anterior position of the RV may render it more susceptible to incomplete myocardial protection than the $\mathrm{LV}^{31}$ Regarding CPB, previous studies in both adults and children have reported that following a variety of cardiac surgeries using $\mathrm{CPB}$, specifically RV performance remained impaired. ${ }^{5,29}$ Furthermore, a negative correlation between CPB time and ventricular performance has been described in patients with congenital heart defect. ${ }^{15,32}$ Finally, a prolonged CPB time was associated with abnormal exercise capacity 13 years after ASO. ${ }^{4}$ The lack of correlation between $\mathrm{CPB}$ and $\mathrm{RV}$ performance parameters observed in our study does not support this suggestion. However, the small sample size and limited spread of CPB times may have concealed a correlation.

Finally, postoperative residua and complications, including pulmonary artery obstruction and coronary artery problems, may continuously alter RV performance. In patients born with TGA, pulmonary artery obstruction is a common complication of the Lecompte procedure, requiring reintervention in $10 \%$ of patients. ${ }^{2}$ A significant pulmonary artery obstruction will increase afterload, which may negatively influence RV performance. ${ }^{26}$ Furthermore, significant pulmonary artery obstruction was a predictor of decreased exercise capacity 13 years postoperatively. ${ }^{4}$ In contrast to these results, no significant correlation between maximal pulmonary artery velocity at discharge and RV performance parameters was observed in our study. Furthermore, at discharge no differences were observed in RV performance parameters between patients with and without significant pulmonary stenosis. However, because pulmonary artery obstruction may develop over time, these results do not exclude a possible influence of pulmonary artery obstructions during more long-term follow-up.

Lastly, ischemic damage caused by coronary artery injury has been suggested to negatively influence postoperative $\mathrm{RV}$ performance. ${ }^{21}$ Damage is possible during transfer and fixation of the coronary arteries. In our study, in only 1 patient was an injury to the coronary arteries described. Still, coronary artery damage frequently goes unnoted during ASO, thus contribution of this factor cannot be excluded based on these results. In addition, a differing coronary artery anatomy, which is associated with TGA, may prolong the surgical procedure. ${ }^{33}$ This may also have a negative influence on cardiac performance. Yet, no significant differences were observed in aortic crossclamp time or CPB time in patients with and without differing coronary artery anatomy. This suggests that the presence of differing coronary artery anatomy does not or minimally influence the duration of $\mathrm{CPB}$. This conclusion is in line with the suggestions by Giardini and colleagues. ${ }^{4}$

\section{Limitations}

TDI parameters have been suggested to be age-dependent. ${ }^{34}$ Hence, the observed changes in TDI parameters preoperatively and 1 day postoperatively versus 1 year postoperatively may in part be age-related changes. Furthermore, special care must be taken in the interpretation of results of changes in TDI parameters preoperatively versus 1 year postoperatively, because these results may also be influenced by the load-dependence of TDI parameters. Nevertheless, similar follow-up is observed in LV global peak strain parameters, which are thought to be less age-dependent. ${ }^{35}$ Finally our main conclusion, the persistent impairment of $\mathrm{RV}$ performance, is not affected by this age-dependency because age-matched controls were used.

The small sample size included in this study prohibited solid analyses of the influence of clinical parameters, including pulmonary artery stenosis, on postoperative impairment of RV performance. Hence, based on our results we cannot rule these out as possible causes for the impaired RV performance.

\section{CONCLUSIONS}

After an initial decline in biventricular performance in patients born with TGA following ASO, LV systolic and diastolic performance recovered to control values within the first postoperative year. Hence, our results emphasize that if early ASO is performed, LV performance is not 
persistently impaired postoperatively. In contrast, RV systolic and diastolic performance remained impaired within the first year following ASO. The cause of the persistent impairment of RV performance may be multifactorial, including direct surgical trauma, reoxygenation injury, CPB-associated ischemia or inflammatory cascades, persistent pulmonary artery obstruction, or damage to the coronary arteries. Our study could not elucidate the exact cause. However, our results do stress the importance of follow-up of RV systolic and diastolic performance following ASO.

The authors thank Gwen C. Bos, Eveline M. Ligthart, Francisca P. Klerk-Voll, and Pytsje D. Stellingwerf for their efforts in performing the echocardiographic studies. The authors also thank Theo Stijnen of the Medical Statistics and Bioinformatics Department, Leiden University Medical Center, for providing statistics support.

\section{References}

1. Sarris GE, Chatzis AC, Giannopoulos NM, Kirvassilis G, Berggren H, Hazekamp M, et al. The arterial switch operation in Europe for transposition of the great arteries: a multi-institutional study from the European Congenital Heart Surgeons Association. J Thorac Cardiovasc Surg. 2006;132:633-9.

2. Lalezari S, Bruggemans EF, Blom NA, Hazekamp MG. Thirty-year experience with the arterial switch operation. Ann Thorac Surg. 2011;92:973-9.

3. van Beek E, Binkhorst M, de Hoog M, de Groot P, van Dijk A, Schokking M, et al. Exercise performance and activity level in children with transposition of the great arteries treated by the arterial switch operation. Am J Cardiol. 2010; 105:398-403

4. Giardini A, Khambadkone S, Rizzo N, Riley G, Pace Napoleone C, Muthialu N, et al. Determinants of exercise capacity after arterial switch operation for transposition of the great arteries. Am J Cardiol. 2009;104:1007-12.

5. Klitsie LM, Kuipers IM, Roest AA, van der Hulst AE, Stijnen T, Hazekamp MG, et al. Disparity in right vs left ventricular recovery during follow-up after ventricular septal defect correction in children. Eur J Cardiothorac Surg. 2013;44:269-74.

6. Bryant RM, Shirley RL, Ott DA, Feltes TF. Left ventricular performance following the arterial switch operation: use of noninvasive wall stress analysis in the postoperative period. Crit Care Med. 1998;26:926-32.

7. Cheung MM, Smallhorn JF, Vogel M, Van Arsdell G, Redington AN. Disruption of the ventricular myocardial force-frequency relationship after cardiac surgery in children: noninvasive assessment by means of tissue Doppler imaging. J Thorac Cardiovasc Surg. 2006;131:625-31.

8. Marcucci C, Lauer R, Mahajan A. New echocardiographic techniques for evaluating left ventricular myocardial function. Semin Cardiothorac Vasc Anesth. 2008; $12: 228-47$

9. Lopez-Candales A, Rajagopalan N, Gulyasy B, Edelman K, Bazaz R. Comparative echocardiographic analysis of mitral and tricuspid annular motion: differences explained with proposed anatomic-structural correlates. Echocardiography. 2007;24:353-9.

10. Bruch C, Grude M, Muller J, Breithardt G, Wichter T. Usefulness of tissue Doppler imaging for estimation of left ventricular filling pressures in patients with systolic and diastolic heart failure. Am J Cardiol. 2005;95:892-5

11. Ng AC, Tran DT, Newman M, Allman C, Vidaic J, Lo ST, et al. Left ventricular longitudinal and radial synchrony and their determinants in healthy subjects. J Am Soc Echocardiogr. 2008;21:1042-8.

12. Klitsie LM, Roest AA, van der Hulst AE, Stijnen T, Blom NA, Ten Harkel AD. Assessment of intraventricular time differences in healthy children using twodimensional speckle-tracking echocardiography. J Am Soc Echocardiogr. 2013;6:629-39.

13. Perk G, Tunick PA, Kronzon I. Non-Doppler two-dimensional strain imaging by echocardiography - from technical considerations to clinical applications. J Am Soc Echocardiogr. 2007;20:234-43.

14. Overbeek LI, Kapusta L, Peer PG, de Korte CL, Thijssen JM, Daniels O. New reference values for echocardiographic dimensions of healthy Dutch children. Eur J Echocardiogr. 2006; 7:113-21.
15. Klitsie LM, Hazekamp MG, Roest AA, van der Hulst AE, Gesink-van der Veer BJ, Kuipers IM, et al. Tissue Doppler imaging detects impaired biventricular performance shortly after congenital heart defect surgery. Pediatr Cardiol. 2013;34:630-8.

16. Corno AF, Milano G, Samaja M, Tozzi P, von Segesser LK. Chronic hypoxia: a model for cyanotic congenital heart defects. J Thorac Cardiovasc Surg. 2002; 124:105-12

17. Drighil A, Madias JE, Mathewson JW, El Mosalami H, El Badaoui N, Ramdani B, et al. Haemodialysis: effects of acute decrease in preload on tissue Doppler imaging indices of systolic and diastolic function of the left and right ventricles. Eur J Echocardiogr. 2008;9:530-5.

18. Hui L, Chau AK, Leung MP, Chiu CS, Cheung YF. Assessment of left ventricular function long term after arterial switch operation for transposition of the great arteries by dobutamine stress echocardiography. Heart. 2005;91:68-72

19. Grotenhuis HB, Ottenkamp J, Fontein D, Vliegen HW, Westenberg JJ, Kroft LJ, et al. Aortic elasticity and left ventricular function after arterial switch operation: MR imaging —initial experience. Radiology. 2008;249:801-9.

20. Colan SD, Boutin C, Castaneda AR, Wernovsky G. Status of the left ventricle after arterial switch operation for transposition of the great arteries. Hemodynamic and echocardiographic evaluation. J Thorac Cardiovasc Surg. 1995;109:311-21.

21. Pettersen E, Fredriksen PM, Urheim S, Thaulow E, Smith HJ, Smevik B, et al Ventricular function in patients with transposition of the great arteries operated with arterial switch. Am J Cardiol. 2009;104:583-9.

22. Vandekerckhove KD, Blom NA, Lalezari S, Koolbergen DR, Rijlaarsdam ME, Hazekamp MG. Long-term follow-up of arterial switch operation with an emphasis on function and dimensions of left ventricle and aorta. Eur J Cardiothorac Surg. 2009;35:582-8.

23. Salzer-Muhar U, Proll E, Marx M, Salzer HR, Wimmer M. Two-dimensional and Doppler echocardiographic follow-up after the arterial switch operation for transposition of the great arteries. Thorac Cardiovasc Surg. 1991;39(Suppl 2):180-4.

24. Taylor AM, Dymarkowski S, Hamaekers P, Razavi R, Gewillig M, Mertens L, et al. MR coronary angiography and late-enhancement myocardial MR in children who underwent arterial switch surgery for transposition of great arteries. Radiology. 2005;234:542-7

25. Martin RP, Qureshi SA, Ettedgui JA, Baker EJ, O'Brien BJ, Deverall PB, et al. An evaluation of right and left ventricular function after anatomical correction and intra-atrial repair operations for complete transposition of the great arteries. Circulation. 1990;82:808-16.

26. Grotenhuis HB, Kroft LJ, van Elderen SG, Westenberg JJ, Doornbos J, Hazekamp MG, et al. Right ventricular hypertrophy and diastolic dysfunction in arterial switch patients without pulmonary artery stenosis. Heart. 2007;93:1604-8

27. Meluzin J, Spinarova L, Hude P, Krejci J, Kincl V, Panovsky R, et al. Prognostic importance of various echocardiographic right ventricular functional parameters in patients with symptomatic heart failure. J Am Soc Echocardiogr. 2005;18 435-44.

28. Chrysohoou C, Antoniou CK, Kotrogiannis I, Metallinos G, Aggelis A, Andreou I, et al. Role of right ventricular systolic function on long-term outcome in patients with newly diagnosed systolic heart failure. Circ J. 2011;75:2176-81.

29. Schuuring MJ, Bolmers PP, Mulder BJ, de Bruin-Bon RA, Koolbergen DR, Hazekamp MG, et al. Right ventricular function declines after cardiac surgery in adult patients with congenital heart disease. Int $J$ Cardiovasc Imaging. 2011:28:755-62

30. Unsworth B, Casula RP, Kyriacou AA, Yadav H, Chukwuemeka A, Cherian A, et al. The right ventricular annular velocity reduction caused by coronary artery bypass graft surgery occurs at the moment of pericardial incision. Am Heart $J$. 2010;159:314-22.

31. Chen YF, Chen JS, Wang JR, Chiu CC, Lin YT. Uneven myocardial hypothermia among cardiac chambers during hypothermic myocardial preservation. Eur $J$ Cardiothorac Surg. 1990;4:618-23.

32. Vassalos A, Lilley S, Young D, Peng E, MacArthur K, Pollock J, et al. Tissue Doppler imaging following paediatric cardiac surgery: early patterns of change and relationship to outcome. Interact Cardiovasc Thorac Surg. 2009;9:173-7.

33. Pasquali SK, Marino BS, McBride MG, Wernovsky G, Paridon SM. Coronary artery pattern and age impact exercise performance late after the arterial switch operation. J Thorac Cardiovasc Surg. 2007;134:1207-12.

34. Eidem BW, McMahon CJ, Cohen RR, Wu J, Finkelshteyn I, Kovalchin JP, et al. Impact of cardiac growth on Doppler tissue imaging velocities: a study in healthy children. J Am Soc Echocardiogr. 2004;17:212-21.

35. Bharucha T, Mertens L. Recent advances in pediatric echocardiography. Expert Rev Cardiovasc Ther. 2013:11:31-47. 